\title{
BOUNDARY REGULARITY OF HARMONIC MAPS TO NONPOSITIVELY CURVED METRIC SPACES
}

\author{
TOMASZ SERBINOWSKI
}

\begin{abstract}
We consider an energy minimizing map from a bounded domain $\Omega$ of a complete, smooth, Riemannian manifold $M^{n}$ to a metric space $X$ of nonpositive curvature. We show regularity of the map near the boundary assuming that its trace is either Lipschitz or Hölder continuous.
\end{abstract}

\section{INTRODUCTION}

This paper was initially motivated by the work of M. Gromov and R. Schoen [G.S.] on $p$-adic superrigidity for lattices in groups of rank one. The main tool in the Gromov-Schoen paper is a theory of harmonic maps into a special class of singular spaces (locally compact Riemannian simplicial complexes embedded into $\mathbf{R}^{n}$ ). They prove existence of a unique Sobolev space solution to the Dirichlet problem and interior Lipschitz continuity of that solution. However, they do not address boundary regularity questions.

More recently N. Korevaar and R. Schoen [K.S.] found a more general way of studying Sobolev spaces and harmonic maps into non-smooth targets. Consequently, they generalized many of the results regarding harmonic map theory from the Gromov-Schoen paper to the case when the target is an arbitrary length space of nonpositive curvature. In particular, they showed existence of a unique solution to the Dirichlet problem for maps into such targets and again, the interior Lipschitz continuity of that solution.

Our goal in the present paper is to prove boundary regularity for solutions to Dirichlet problem, in the generality of the Korevaar-Schoen work.

Throughout this paper $(X, d)$ will denote a complete metric space and 
$\Omega^{n}$ will be a bounded domain in a smooth, complete, Riemannian manifold $\left(M^{n}, g\right)$.

Definition. We say that a Borel-measurable map $u:(\Omega, g) \rightarrow(X, d)$ is in $L^{2}(\Omega, X)$ if it has separable range and for some point $Q \in X$

$$
\int_{\Omega} d^{2}(u(x), Q) d \mu_{g}(x)<\infty
$$

The set $L^{2}(\Omega, X)$ is a complete metric space (see e.g. [K.S.], $§ 1.1$ ) with the distance function defined by

$$
D(u, v)=\left(\int_{\Omega} d^{2}(u(x), v(x)) d \mu_{g}(x)\right)^{\frac{1}{2}} .
$$

$L^{2}(\Omega, X)$ contains a subset $W^{1,2}(\Omega, X)$ consisting of finite energy maps. The energy of a map $u \in L^{2}(\Omega, X)$ is defined as follows (after [K.S.], $\S \S 1$., $1.3)$.

First we observe that for every $\epsilon>0$

$$
e_{\epsilon}(x)=n\left|\partial B_{\epsilon}(x)\right|^{-1} \int_{\partial B_{\epsilon}(x)} \frac{d^{2}(u(x), u(y))}{\epsilon^{2}} d \Sigma
$$

is well defined for almost all $x \in \Omega$ (if $\operatorname{dist}(x, \partial \Omega)<\epsilon$ then let $e_{\epsilon}(x)=0$ ). Moreover, $e_{\epsilon}(x) \in L^{1}(\Omega)$, and therefore it defines a functional $E_{\epsilon}$ on the space of compactly supported functions $C_{c}(\Omega)$ (via integration).

Definition. ([K.S.], 1.3ii) The map $u \in L^{2}(\Omega, X)$ is said to have finite energy if

$$
\sup _{\substack{f \in C_{c}(\Omega) \\ 0 \leq f \leq 1}}\left(\limsup _{\epsilon \rightarrow 0} E_{\epsilon}(f)\right) \equiv E<\infty
$$

If $u$ has finite energy then in fact there exists a function $e(x) \in L^{1}(\Omega)$ so that $e_{\epsilon}(x) d \mu_{g}(x) \rightarrow e(x) d \mu_{g}(x)$ as measures. In particular

$$
E=\int_{\Omega} e(x) d \mu_{g}(x)
$$

The function $e(x)$ is called the energy density of $u$, and $E$ is defined to be the (total) energy of $u$. 
For two finite energy maps $u$ and $v$ the distance $d(u, v): \Omega \rightarrow \mathbf{R}_{+}$between them belongs to the Sobolev space $W^{1,2}(\Omega)$ ([K.S.], 1.6vi). Therefore one can make the following definition

$$
u=v \text { on } \partial \Omega \text { if } d(u, v) \in W_{0}^{1,2}(\Omega) .
$$

Definition. We say that a finite energy map $u: \Omega \rightarrow X$ is energy minimizing if

$$
E^{u}=\inf \left\{E^{v}: v \text { has finite energy, } v=u \text { on } \partial \Omega\right\} .
$$

In this paper we want to study boundary regularity of energy minimizing maps in the case when the metric space $X$ satisfies some additional conditions.

Consider a continuous path $\gamma:[0,1] \rightarrow X$ joining two points $P=\gamma(0)$ and $Q=\gamma(1)$. Obviously $d(P, Q) \leq L(\gamma)=$ length $(\gamma)$.

Definition. The metric space $(X, d)$ is called a length space if for every pair of points $P, Q \in X$ there exists a distance realizing path $\gamma:[0,1] \rightarrow X$, that is a path satisfying $\gamma(0)=P, \gamma(1)=Q$, and $L(\gamma)=d(P, Q)$. Observe that every such distance realizing path can be parametrized proportionally to the length, and then

$$
d(\gamma(0), \gamma(t))=t d(\gamma(0), \gamma(1)) .
$$

Distance realizing paths parametrized proportionally to length are called geodesics.

Definition. (cf. [G.S.]) We say that a length space $(X, d)$ has nonpositive curvature if for every geodesic $\gamma:[0,1] \rightarrow X$ and point $Q \in X$

$$
d^{2}(\gamma(t), Q) \leq t d^{2}(\gamma(1), Q)+(1-t) d^{2}(\gamma(0), Q)-t(1-t) d^{2}(\gamma(0), \gamma(1)) .
$$

Geometrically this means that if we compare a geodesic triangle in $X$ to a triangle in the Euclidean plane with the same side lengths, then corresponding secants are longer for the Euclidean one.

Let $(X, d)$ be a length space of nonpositive curvature and let $\Omega$ be a bounded Lipschitz Riemannian domain. Let $\phi: \Omega \rightarrow X$ be a given finite energy map, $\phi \in W^{1,2}(\Omega, X)$. Korevaar-Schoen showed that there exists a unique, finite energy map $u: \Omega \rightarrow X$ satisfying

(i) $E^{u}=\inf \left\{E^{v}: v\right.$ has finite energy, $v=\phi$ on $\left.\partial \Omega\right\}$ 
(ii) $u=\phi$ on $\partial \Omega$.

([K.S.], Theorem 2.2).

In particular the map $u$ is energy minimizing. Furthermore, they proved that this map $u$ is Lipschitz continuous in the interior of $\Omega$ ([K.S.], Theorem 2.4.6). In case $X$ is a Riemannian simplicial complex isometrically embedded in Euclidean space, this theorem was proven by Gromov-Schoen ([G.S.], Lemma 1.1 and Theorem 2.3).

Though Korevaar-Schoen in their paper work only with Lipschitz domains (and develop trace theory for $W^{1,2}$ maps), the above existence result holds for an arbitrary (non-Lipschitz) domain $\Omega$.

Namely, if $\left\{u_{i}\right\} \subset W^{1,2}(\Omega, X)$ is a sequence such that

$$
d\left(u_{i}, \phi\right) \in W_{0}^{1,2}(\Omega)
$$

and

$$
E^{u_{i}} \searrow E_{0}=\inf \left\{E^{v}: v \text { has finite energy and } d(v, \phi) \in W_{0}^{1,2}(\Omega)\right\}
$$

then from energy convexity ([K.S.], 2.2iv)

$$
\lim _{i, j \rightarrow \infty} \int_{\Omega}\left|\nabla d\left(u_{i}, u_{j}\right)\right|^{2} \lambda_{\prime \prime}=0
$$

Therefore (by Poincare inequality) $\left\{u_{i}\right\} \subset L^{2}(\Omega, X)$ is a Cauchy sequence with uniformly bounded energies, and hence $u_{i} \stackrel{L^{2}}{\rightarrow} u \in W^{1,2}(\Omega, X)$ ([K.S.], Theorem 1.6.1). What must be checked is that $d(u, \phi) \in W_{0}^{1,2}(\Omega)$.

From the triangle inequality

$$
\begin{aligned}
\mid d\left(u(x), u_{i}(x)\right)- & d\left(u(y), u_{i}(y)\right) \mid \\
\leq d\left(u(x), u_{i+k}(x)\right) & +\left|d\left(u_{i}(x), u_{i+k}(x)\right)-d\left(u_{i}(y), u_{i+k}(y)\right)\right| \\
& +d\left(u(y), u_{i+k}(y)\right)
\end{aligned}
$$

we deduce that for any fixed $\epsilon>0$

$$
E_{\epsilon}^{d\left(u, u_{i}\right)} \leq \frac{6 n}{\epsilon^{2}}\left\|d\left(u, u_{i+k}\right)\right\|_{L^{2}}^{2}+3\left\|\nabla d\left(u_{i}, u_{i+k}\right)\right\|_{L^{2}}^{2} .
$$

In particular, for given $\delta>0$, by choosing $i$ large enough to have

$$
\left\|\nabla d\left(u_{i}, u_{i+k}\right)\right\|_{L^{2}}^{2}<\delta
$$


and letting $k \rightarrow 0$, we get that $\epsilon$-energy of $d\left(u, u_{i}\right)$ is bounded by $3 \delta$. Consequently, $E^{d\left(u, u_{i}\right)} \rightarrow 0$ and therefore $d\left(u, u_{i}\right) \rightarrow 0$ in $W^{1,2}(\Omega)$.

Since $\left|d(u, \phi)-d\left(u, u_{i}\right)\right| \leq d\left(u_{i}, \phi\right) \in W_{0}^{1,2}(\Omega)$ and $d(u, \phi)=\lim _{i \rightarrow \infty}(d(u, \phi)-$ $\left.d\left(u, u_{i}\right)\right)$ we conclude that $d(u, \phi) \in W_{0}^{1,2}(\Omega)$ and hence $u$ is an energy minimizing map.

We will prove that the energy minimizing function $u$ is Hölder continuous near the boundary $\partial \Omega$, assuming that its trace is so (meaning $d(u, \phi) \in$ $W_{0}^{1,2}(\Omega)$ for a Hölder continuous map $\left.\phi\right)$. The modulus of the continuity of $u$, roughly speaking, depends on the regularity of the trace of $u$ and on the "regularity" of the boundary $\partial \Omega$ itself. The latter will be formulated in terms of local exterior cones.

Definitions. For a point $p \in M$ and a unit vector $V \in T_{p} M(|V|=1)$, we define an (open) $\vartheta$-cone at $p$ in direction $V$ as

$$
C_{\vartheta}^{\sigma}(p, V)=\left\{q=\exp _{p}(s W): 0<s<\sigma,|W|=1, g(V, W)>\cos \vartheta\right\}
$$

If $p \in \partial \Omega$ then for each $\sigma>0$ we set $B_{\sigma}^{+}(p)=B_{\sigma}(p) \cap \Omega$ and $S_{\sigma}^{+}(p)=S_{\sigma}(p) \cap \Omega$. Furthermore by $\vartheta_{p}(\sigma)$ we denote an angle of the biggest exterior cone at $p$. More precisely, $\vartheta_{p}(\sigma)$ is the biggest $\vartheta \in[0, \pi / 2]$ such that for some unit vector $V \in T_{p} M$ an $\vartheta$-cone locally lies outside $\Omega$

$$
C_{\vartheta}^{\sigma}(p, V) \cap \Omega=\emptyset
$$

Note that $\vartheta_{p}(\sigma)$ is monotone. Finally we introduce the quantity

$$
m_{p}(\sigma)=\frac{\left[\sin \left(\pi-\vartheta_{p}(\sigma)\right)\right]^{n-2}}{\left(\pi-\vartheta_{p}(\sigma)\right)^{n}}\left(\frac{\pi}{2}\right)^{n}
$$

We remark here that for a Lipschitz domain, there is always a uniform lower bound on the angle $\vartheta_{p}(\sigma)$, and hence on $m_{p}(\sigma)$. If $\partial \Omega$ has a tangent plane at $p$, then $m_{p}(\sigma) \rightarrow 1$ as $\sigma \searrow 0$.

The results of this paper are:

Theorem. Suppose that $\phi:\left(\Omega^{n}, g\right) \rightarrow(X, d)$ is a finite energy map from a bounded domain in $M$ to a length space of nonpositive curvature. Let $u$ : $\left(\Omega^{n}, g\right) \rightarrow(X, d)$ be the energy minimizing map such that $u=\phi$ on $\partial \Omega$. 
Assume that for relatively open subset $\Gamma \subset \partial \Omega$ we have $\phi \in C_{\text {loc }}^{\alpha}(\Omega \cup \Gamma)$. Let $m>0$ be (the biggest) constant such that for all $p \in \Gamma$

$$
m_{p}(\sigma) \geq m-O(\sigma)
$$

(with $O(\sigma)$ uniform in $p$ ). Then

(A) $\alpha=m \Longrightarrow u \in C_{l o c}^{\beta}(\Omega \cup \Gamma)$ for every $0 \leq \beta<\alpha$

(B) $\alpha \neq m \Longrightarrow u \in C_{l o c}^{\beta}(\Omega \cup \Gamma)$ for $\beta=\min (\alpha, m)$.

Remark. The estimates for the Hölder exponents are not sharp. This is due to the fact that $m_{p}(\sigma)$ is not the best constant in (9). In the case $n=2$, using conformal change of coordinates one shows that (9) holds with $\tilde{m}_{p}(\sigma)=\frac{\pi}{2(\pi-\vartheta)}$, and it is easy to check that the resulting estimates are sharp. (Consider harmonic function $u(z)=\Re\left(z^{\alpha}\right)$ on $\Omega=\{|\arg (z)|<\pi / 2 \alpha\}$.)

Corollary. Suppose that $\phi:\left(\Omega^{n}, g\right) \rightarrow(X, d)$ is a finite energy map from a smooth bounded domain in $M$ into a length space of nonpositive curvature. Let $u:\left(\Omega^{n}, g\right) \rightarrow(X, d)$ be an energy minimizing map such that $u=\phi$ on $\partial \Omega$. Then

(A) $\phi \in \operatorname{Lip}(\bar{\Omega}) \Longrightarrow u \in C^{\beta}(\bar{\Omega})$, for every $0 \leq \beta<1$

(B) $\phi \in C^{\alpha}(\bar{\Omega}) \Longrightarrow u \in C^{\alpha}(\bar{\Omega})$.

Proof. The Corollary follows immediately from the observation that in this case we have a uniform bound $\vartheta_{p}(\sigma) \geq \frac{\pi}{2}-O(\sigma)$ and consequently $m_{p}(\sigma) \geq$ $1-O(\sigma)$.

\section{PREliminary Lemmas}

In our proof, generally, we intend to build on the ideas of Gromov-Schoen. However, we will use techniques of Korevaar-Schoen to carry out the arguments in needed generality.

The Gromov-Schoen proof of interior regularity depends on two specific families of variations (domain variations, range variations) and the resulting estimates. Below we summarize relevant parts of their proof.

For a point $Q \in X$ they consider the function $x \longmapsto d^{2}(u(x), Q)$ (note that $\left.d^{2}(u(x), Q) \in W^{1,2}(\Omega)\right)$. Their range variations (which use essentially the 
nonpositivity of the curvature of $X$ ) lead to the (weak) differential inequality ([G.S.], Proposition 2.2)

$$
\Delta d^{2}(u(x), Q)-2|\nabla u|^{2} \geq 0 .
$$

(In particular, the functions $d^{2}(u(x), Q)$ and even $d(u(x), Q)$ are (weakly) subharmonic.) Consequently, for almost all $\sigma>0$ they deduce

$$
2 \int_{B_{\sigma}}|\nabla u|^{2} d \mu_{g}(x) \leq \int_{\partial B_{\sigma}} \frac{\partial}{\partial r} d^{2}(u, Q) d \Sigma .
$$

Using domain variations they show that ([G.S.], 2.3)

$0=\left(2-n+O\left(\sigma^{2}\right)\right) \int_{B_{\sigma}(p)}|\nabla u|^{2} d \mu_{g}(x)+\sigma \int_{\partial B_{\sigma}(p)}|\nabla u|^{2} d \Sigma-2 \sigma \int_{\partial B_{\sigma}(p)}\left|\frac{\partial u}{\partial r}\right|^{2} d \Sigma$.

The rest of their argument for interior Lipschitz continuity (which we will not repeat) involves differential inequalities for

$$
E(\sigma)=\int_{B_{\sigma}(p)}|\nabla u|^{2} d \mu_{g}(x), \quad I(\sigma)=\int_{\partial B_{\sigma}(p)} d^{2}(u, Q) d \Sigma,
$$

which follow from (1), (2), (3), and Calculus.

One further conclusion (see the differential inequality for $I(\sigma)$ in the proof of [G.S.] Theorem 2.3) which we will need is that for $Q=u(x)$

$$
\sigma \longmapsto \frac{1+O\left(\sigma^{2}\right)}{\sigma^{n+1}} \int_{\partial B_{\sigma}(x)} d^{2}(u(y), Q) d \Sigma \quad \text { is nondecreasing. }
$$

Korevaar-Schoen remark that (1), (2), (3) and hence their consequences generalize to nonpositively curved length spaces $X$. They follow a different approach in showing regularity, however, and do not supply in [K.S.] the details for this claim. We note here that the consequences of domain variations (3) follows exactly as in ([G.S.], §2) using ([K.S.], Theorem 2.3.2) to justify various steps. The subharmonicity statement (1) (hence also (2)) follows from triangle comparisons, and we present an argument of Korevaar-Schoen which they did not include in [K.S.].

As $X$ is assumed to have nonpositive curvature, there is a unique geodesic joining any given pair of points $P, Q \in X$. Therefore, given $Q \in X$ and $\lambda \in$ $[0,1]$ one can define a map $R_{\lambda}: X \rightarrow X$ via the formula

$R_{\lambda}(P)=\psi(\lambda)$, where $\psi(s)$ is unique geodesic with $\psi(0)=Q$ and $\psi(1)=P$. 
The maps $R_{\lambda}$ are Lipschitz continuous and satisfy $d\left(R_{\lambda}\left(P_{1}\right), R_{\lambda}\left(P_{2}\right)\right) \leq \lambda d\left(P_{1}, P_{2}\right)$.

Let $\eta \in C_{c}^{\infty}(\Omega), \eta \geq 0$. For $t \geq 0$ we consider a finite energy map $u_{t}(x)=$ $R_{1-t \eta(x)}(u(x))$. As $\eta=0$ on $\partial \Omega$ it follows that $u_{t}=u=\phi$ on $\partial \Omega$. Therefore $E^{u_{t}} \geq E^{u}$.

If we can show that

$$
E^{u_{t}} \leq E^{u}-t \int_{\Omega}\left[\nabla \eta \bullet \nabla d^{2}(u, Q)+2 \eta e(x)\right]+O\left(t^{2}\right)
$$

then, by letting $t \rightarrow 0$, we deduce the weak version of (1)

$$
-\int_{\Omega} \nabla \eta \bullet \nabla d^{2}(u, Q) \geq 2 \int_{\Omega} \eta e(x), \quad \text { for any } \eta \in C_{c}^{\infty}(\Omega), \eta \geq 0 .
$$

Inequality (5) follows from the distance comparisons as follows . For a pair of points $x, y \in \Omega$ we consider a geodesic triangle in $X$ with vertices $u(x), u(y)$, and $Q$. Note that points $u_{t}(x)$ and $u_{t}(y)$ lie on the triangle sides. Moreover

$$
d\left(u_{t}(x), Q\right)=(1-t \eta(x)) d(u(x), Q), \quad d\left(u_{t}(y), Q\right)=(1-t \eta(y)) d(u(y), Q) .
$$

A consequence of the nonpositive curvature condition is that $d\left(u_{t}(x), u_{t}(y)\right)$ is bounded above by the distance between the corresponding points of the Euclidean triangle with side lengths $d(u(x), Q), d(u(y), Q), d(u(x), u(y))$. By explicit computation this yields

$$
\begin{aligned}
& d^{2}\left(u_{t}(x), u_{t}(y)\right) \\
\leq & (1-t \eta(x))^{2} d^{2}(u(x), Q)+(1-t \eta(y))^{2} d^{2}(u(y), Q) \\
& +(1-t \eta(x))(1-t \eta(y))\left[d^{2}(u(x), u(y))-d^{2}(u(x), Q)-d^{2}(u(y), Q)\right] \\
= & d^{2}(u(x), u(y))-t\left[(\eta(x)+\eta(y)) d^{2}(u(x), u(y))\right. \\
& \left.+(\eta(x)-\eta(y))\left(d^{2}(u(x), Q)-d^{2}(u(y), Q)\right)\right]+O\left(t^{2}\right) .
\end{aligned}
$$

As the definition of the energy is based on averaging distances, this estimate and ([K.S.],1.6.2) imply inequality (5), hence (1).

The following estimate is an adaptation of (1) for boundary estimate.

Let $K$ be a compact subset of $\Omega \cup \Gamma(K \subset \subset \Omega \cup \Gamma)$. Denote by $K^{\sigma}$ a $\sigma$-neighborhood of $K$ in $\bar{\Omega}$

$$
K^{\sigma}=\{x \in \bar{\Omega}: \operatorname{dist}(x, K) \leq \sigma\} .
$$


Note that $K^{\sigma}$ is also a compact set. As $\Gamma \subset \partial \Omega$ is assumed to be relatively open, there exists $\sigma_{0}>0$, such that $K^{\sigma_{0}} \subset \subset \Omega \cup \Gamma$. Therefore (because of assumption that $\left.\phi \in C_{l o c}^{\alpha}(\Omega \cup \Gamma)\right)$

$$
c_{K}=[\phi]_{\alpha, K^{\sigma_{0}}}=\sup _{x, y \in K^{\sigma_{0}}} \frac{d(\phi(x), \phi(y))}{|x-y|^{\alpha}}<\infty .
$$

Let $p \in \Gamma \cap K, Q=\phi(p)$, and $d(x)=d(u(x), Q)$. For $\sigma<\frac{1}{2} \sigma_{0}$ we define $d_{\sigma}=\left(d-2 c_{K} \sigma^{\alpha}\right)^{+}$.

The following lemma is a consequence of (6).

Lemma 1. Let $\eta$ be a non-negative Lipschitz function with support supp $\eta \subset \subset$ $\Omega \cup \overline{B_{2 \sigma}^{+}(p)}$. Then

$$
-\int_{\Omega} \nabla \eta \bullet \nabla d_{\sigma}^{2} \geq 2 \int_{\Omega} \eta\left|\nabla d_{\sigma}\right|^{2}
$$

Proof. The fact that $d(u, \phi) \in W_{0}^{1,2}(\Omega)$ together with the inequality

$$
0 \leq d_{\sigma}(x) \leq d(u(x), \phi(x)) \quad \text { for } x \in B_{2 \sigma}^{+}(p)
$$

implies that $d_{\sigma} \equiv 0$ on $B_{2 \sigma}(p) \cap \partial \Omega$ (that is, $\psi d_{\sigma} \in W_{0}^{1,2}(\Omega)$ for all $\psi \in$ $\left.C_{c}^{1}\left(\Omega \cup \overline{B_{2 \sigma}^{+}}\right)\right)$. In particular $d_{\sigma}$ can be approximated in $W^{1,2}$-sense by $C^{1}$ functions vanishing on the neighborhood of $B_{2 \sigma}(p) \cap \partial \Omega$, and consequently can be extended by zero across the boundary of $\Omega$.

Since inequality (1) implies weak subharmonicity of $d$ in $\Omega$, it follows that $d_{\sigma}$ is subharmonic as well. Because $d_{\sigma}$ has finite integral over $\Omega$ and $d_{\sigma} \equiv 0$ on $B_{2 \sigma}(p) \cap \partial \Omega$, we can conclude that $d_{\sigma}$ is bounded on the neighborhood of supp $\eta$ (that in turn shows boundedness of $d \leq d_{\sigma}+2 c_{K} \sigma^{\alpha}$ ).

From $\eta$ we construct the auxiliary test function

$$
\zeta=\eta \frac{d_{\sigma}}{d}=\eta \frac{d_{\sigma}}{\max \left(d, 2 c_{K} \sigma^{\alpha}\right)} .
$$

Since $d_{\sigma}$ is zero on $B_{2 \sigma}(p) \cap \partial \Omega$, and $\eta$ is zero on $\partial \Omega \backslash B_{2 \sigma}(p)$, it is also true that $\zeta \in W_{0}^{1,2}(\Omega)$. These two facts and standard approximation arguments imply that $\zeta$ is an admissible test function in (6). Thus

$$
-\int_{\Omega} \nabla\left(\eta \frac{d_{\sigma}}{d}\right) \bullet \nabla d^{2} \geq 2 \int_{\Omega}\left(\eta \frac{d_{\sigma}}{d}\right) e(x) .
$$


By simple manipulations, one can rewrite this inequality as

$$
-\int_{\Omega} \nabla \eta \bullet \nabla d_{\sigma}^{2} \geq 2 \int_{\Omega} \eta\left[\frac{d_{\sigma}}{d} e(x)+\left(1-\frac{d_{\sigma}}{d}\right)\left|\nabla d_{\sigma}\right|^{2}\right] .
$$

Inequality (7) follows from the observation that $e(x) \geq|\nabla d|^{2}$.

By taking $\eta$ to approximate the characteristic function of $B_{\sigma}^{+}$in (7), we obtain a boundary analog of (2) (for almost all sufficiently small $\sigma$ ):

$$
\int_{S_{\sigma}^{+}} \frac{\partial}{\partial r} d_{\sigma}^{2} \geq 2 \int_{B_{\sigma}^{+}}\left|\nabla d_{\sigma}\right|^{2} .
$$

(Existence of the left-hand-side integral follows from the absolute continuity of $\sigma \longmapsto \int_{S_{\sigma}^{+}} d_{\sigma}^{2}$.)

We shall also use the following Calculus estimate, which allows us to pursue boundary regularity without an exact boundary analog of (3).

If $f \in W^{1,2}\left(B_{\sigma}^{+}\right)$satisfies $f \equiv 0$ on $\partial \Omega \cap B_{\sigma}(p)$ then

$$
\sigma \int_{B_{\sigma}^{+}}|\nabla f|^{2} \geq\left(m_{p}(\sigma)-O\left(\sigma^{2}\right)\right) \int_{S_{\sigma}^{+}} f^{2}
$$

where $O\left(\sigma^{2}\right)$ depends on $\Omega$ and $g$ (but not on function $f$ ).

It suffices to prove (9) for a smooth function $f \in C^{\infty}\left(\overline{B_{\sigma}^{+}}\right)$which vanishes $(f \equiv 0)$ on the neighborhood of $\partial \Omega \cap B_{\sigma}(p)$. Choose $x=\left(x_{1}, \ldots, x_{n}\right)$ to be the geodesic normal coordinates centered at $p$. By definition of $\vartheta_{p}(\sigma)$ there exist a cone $C=C_{\vartheta_{p}(\sigma)}^{\sigma}(p, V)$ so that $C \cap \Omega=\emptyset$. Without loss of the generality we can assume that $V=-\frac{\partial}{\partial x_{n}}$. Further we may extend the function $f$ by zero to all of $B_{\sigma}(p)$. Then we compute

$$
\int_{B_{\sigma}^{+}}\left|\nabla_{g} f\right|^{2} d \mu_{g}(x)=\left(1+O\left(\sigma^{2}\right)\right) \int_{B_{\sigma} \backslash C}\left|\nabla_{x} f\right|^{2} d x
$$

where $\left|\nabla_{x} f\right|^{2}=\sum\left(\frac{\partial f}{\partial x_{i}}\right)^{2}$ and $d x=d x_{1} \ldots d x_{n}$, are Euclidean gradient and volume form.

Let $D=\left\{y:|y| \leq \sigma\right.$ and $\left.y_{n}>0\right\}$ and $D^{\prime}=D \cap\{|y|=\sigma\}$. On $D$ we introduce spherical coordinates $(r, \omega, \theta)$. For $y=\left(y^{\prime}, y_{n}\right)$ we have

$$
|y|=r, \quad y^{\prime}=r \omega \sin \theta, \quad y_{n}=r \cos \theta .
$$


We define one-to-one Lipschitz map $h: D \longrightarrow B_{\sigma} \backslash C$ via

$$
h(y)=h\left(y^{\prime}, y_{n}\right)=(r \omega \sin (a \theta), r \cos (a \theta))=\left(x^{\prime}, x_{n}\right)=x
$$

where $a=2-\frac{2}{\pi} \vartheta_{p}(\sigma)$.

One verifies that the Jacobian of $h$ and operator norm of $d h$ are estimated as follows

i) $a\left(\sin a \frac{\pi}{2}\right)^{n-2} \leq|J(h)| \leq a^{n-1}$

ii) $\|d h\|=a$

Therefore integration by substitution yields:

$$
\begin{aligned}
\int_{B_{\sigma} \backslash C}\left|\nabla_{x} f\right|^{2} d x & =\int_{D}\left(\left|\nabla_{x} f\right|^{2} \circ h\right)|J(h)| d y \\
& \geq \int_{D}\left|\nabla_{y}(f \circ h)\right|^{2}\|d h\|^{-2}|J(h)| d y \\
& \geq a^{-1}\left(\sin a \frac{\pi}{2}\right)^{n-2} \int_{D}\left(\left|\nabla_{y}(f \circ h)\right|^{2} d y\right.
\end{aligned}
$$

Using the fundamental theorem of calculus to integrate in $y_{n}$ direction (and noting that $f \circ h \equiv 0$ on $\left\{y_{n}=0\right\}$ ) one sees that

$$
\sigma \int_{D}\left|\nabla_{y}(f \circ h)\right|^{2} d y \geq \int_{D^{\prime}}(f \circ h)^{2} d \Sigma_{y}
$$

Finally (again integrating by substitution) we find that

$$
\begin{aligned}
\int_{D^{\prime}}(f \circ h)^{2} d \Sigma_{y} & =\int_{S_{\sigma}^{+}} f^{2}|J(h)|^{-1} d \Sigma_{x} \\
& \geq a^{1-n} \int_{S_{\sigma}^{+}} f^{2} d \Sigma_{x} \\
& =a^{1-n}\left(1+O\left(\sigma^{2}\right)\right) \int_{S_{\sigma}^{+}} f^{2} d \Sigma_{g}(x)
\end{aligned}
$$

Altogether we get

$$
\sigma \int_{B_{\sigma}^{+}}\left|\nabla_{g} f\right|^{2} d \mu_{g}(x) \geq a^{-n}\left(\sin a \frac{\pi}{2}\right)^{n-2}\left(1-O\left(\sigma^{2}\right)\right) \int_{S_{\sigma}^{+}} f^{2} d \Sigma_{g}(x)
$$

This completes the proof of $(9)$ as $m_{p}(\sigma)=\frac{\left(\sin a \frac{\pi}{2}\right)^{n-2}}{a^{n}}$.

Combining (8) and (9) yields

$$
\sigma \int_{S_{\sigma}^{+}} \frac{\partial}{\partial r} d_{\sigma}^{2} \geq 2\left(m_{p}(\sigma)-O\left(\sigma^{2}\right)\right) \int_{S_{\sigma}^{+}} d_{\sigma}^{2} .
$$




\section{Proof of the Boundary REgularity}

The strategy is to use the differential inequality (10) to deduce a Hölder estimate for $d(u(x), u(y))$ when either $x$ or $y$ is a boundary point. Then, we employ this estimate and the interior machinery developed in [G.S.] (which we summarized in §2), to prove full Hölder continuity of $u$.

Our notation is as in Lemma 1. That is, $K$ is a compact subset of $\Omega \cup \Gamma$, $\sigma_{0}>0$ is such that $K^{\sigma_{0}} \subset \subset \Omega \cup \Gamma$ (note that we can assume $\sigma_{0}<1$ ), $c_{K}=[\phi]_{\alpha, K^{\sigma_{0}}}$, and for a given point $p \in K \cap \Gamma$ we let

$$
d_{\sigma}(x)=\left[d(u(x), \phi(p))-2 c_{K} \sigma^{\alpha}\right]^{+} .
$$

For clarity, we present the proof only in case $\alpha \neq m$. Let then $\beta=\min (\alpha, m)$, and let $I(\sigma)=\int_{S_{\sigma}^{+}(p)} d_{\sigma}^{2}(x) d \Sigma$. All the constants in the lemmas below $\left(c_{1}, c_{2}, \ldots\right)$ depend on metric $g$, restriction of $\phi$ to $K^{\sigma_{0}}$ (in particular on $c_{K}$ ), bound on $\sup _{K^{\sigma_{0}}} d$, and finally on $\sigma_{0}$. It is crucial that they do not depend on the point $p \in K \cap \Gamma$.

Lemma 2. For some positive constants $c_{1}, c_{2}$ the expression

$$
e^{c_{1} \sigma} \sqrt{\frac{I(\sigma)}{\sigma^{n-1+2 m}}}+c_{2} \frac{\sigma^{\alpha-m}}{\alpha-m} \quad \text { is increasing in } \sigma \text {. }
$$

Proof. First we compute the derivative of $I(\sigma)$. Using Calculus,

$$
I^{\prime}(\sigma)=\left(\frac{n-1}{\sigma}+O(\sigma)\right) I(\sigma)+\int_{S_{\sigma}^{+}} \frac{\partial}{\partial r} d_{\sigma}^{2}-4 c_{K} \alpha \sigma^{\alpha-1} \int_{S_{\sigma}^{+}} d_{\sigma} .
$$

We can use (10) on the second term and the Hölder inequality on the third one to get the differential inequality below

$$
I^{\prime}(\sigma) \geq\left(\frac{n-1+2 m}{\sigma}-O(1)\right) I(\sigma)-c(1+O(\sigma)) \sigma^{\alpha+\frac{n}{2}-\frac{3}{2}} \sqrt{I(\sigma)},
$$

which (after division by $\sqrt{I(\sigma)}$ ) formally yields the differential inequality for $f=\sqrt{I(\sigma)}$ :

$$
f^{\prime}(\sigma) \geq\left(\frac{n-1+2 m}{2 \sigma}-O(1)\right) f(\sigma)-\frac{c}{2}(1+O(\sigma)) \sigma^{\frac{n-3+2 \alpha}{2}} .
$$

Hence for $c_{1}$ determined by the $O(1)$ term ,

$$
\left(e^{c_{1} \sigma} \sigma^{-\frac{n-1+2 m}{2}} f\right)^{\prime} \geq-\frac{c}{2} e^{c_{1} \sigma}(1+O(\sigma)) \sigma^{\alpha-m-1} .
$$


Hence for $c_{2}$ depending on $c, c_{1}$, and the $O(\sigma)$ term

$$
\left(e^{c_{1} \sigma} \sigma^{-\frac{n-1+2 m}{2}} f+c_{2} \frac{\sigma^{\alpha-m}}{\alpha-m}\right)^{\prime} \geq 0 .
$$

Because $f$ is an absolutely continuous function of $\sigma$, the conclusion of the lemma can be derived from that formal differential inequality by considering the open intervals on which $f$ is positive.

Lemma 3. There exists a constant $c_{6}$ such that for all $\sigma<\frac{1}{2} \sigma_{0}$

$$
d \leq c_{6} \sigma^{\beta} \text { on a ball } B_{\sigma}^{+}
$$

Proof. Let $\sigma \leq \sigma_{0}$. Lemma 2 implies

$$
\begin{aligned}
\sqrt{\frac{1}{\sigma^{n-1}} I(\sigma)} & \leq e^{-c_{1} \sigma}\left(c_{3} \sigma^{m}-c_{2} \frac{\sigma^{\alpha}}{\alpha-m}\right) \leq c_{3} \sigma^{m}-c_{2} \frac{\sigma^{\alpha}}{\alpha-m} \\
& \leq\left(c_{3}+\frac{c_{2}}{|\alpha-m|}\right) \sigma^{\beta}=c_{4} \sigma^{\beta}
\end{aligned}
$$

where

$$
c_{3}=e^{c_{1} \sigma_{0}} \sqrt{\frac{I\left(\sigma_{0}\right)}{\sigma_{0}^{n-1+2 m}}}+c_{2} \frac{\sigma_{0}^{\alpha-m}}{\alpha-m} .
$$

From lemma 1 follows immediately that (if extended by zero) $d_{\sigma}^{2}$ is (weakly) subharmonic on the whole ball $B_{\sigma}$ and hence

$$
\sup _{B_{\sigma / 2}^{+}} d_{\sigma}^{2} \leq c_{5} \frac{1}{\sigma^{n-1}} I(\sigma) .
$$

Finally as $d \leq d_{\sigma}+2 c_{K} \sigma^{\alpha}$ we obtain that $d \leq \sqrt{c_{5}} c_{4} \sigma^{\beta}+2 c_{K} \sigma^{\alpha}$ on $B_{\frac{\sigma}{2}}^{+}$, and for $c_{6}=2^{\beta}\left(\sqrt{c_{5}} c_{4}+2 c_{K}\right)$ the conclusion of the lemma holds.

Lemma 3 states that

$$
d\left(u(x), u\left(x_{0}\right)\right) \leq c_{6}\left|x-x_{0}\right|^{\beta}
$$

whenever $x_{0} \in K \cap \Gamma$ and $\left|x-x_{0}\right|<\frac{1}{2} \sigma_{0}$.

Let $\sigma^{\prime}=\sigma_{0} / 2$ and let $K^{\prime}=K^{\sigma^{\prime}}$. Then $K^{\prime}$ is a compact subset of $\Omega \cup \Gamma$. Moreover $\left(K^{\prime}\right)^{\sigma^{\prime}}=K^{\sigma_{0}}$. Therefore for some constant $c_{7}$

$$
d\left(u(x), u\left(x_{0}\right)\right) \leq c_{7}\left|x-x_{0}\right|^{\beta} \text { if } x_{0} \in K^{\prime} \cap \Gamma \text { and }\left|x-x_{0}\right|<\frac{1}{2} \sigma^{\prime} .
$$

(We cannot take $c_{7}$ to be equal to $c_{6}$ because $\sigma^{\prime} \neq \sigma_{0}$.) 
Let $x \in K$ and assume that $\operatorname{dist}(x, \partial \Omega)<\frac{1}{4} \sigma_{0}$. Then there exists point $x_{0} \in \partial \Omega$ such that $\left|x-x_{0}\right|<\frac{1}{4} \sigma_{0}$. In particular $x_{0} \in K^{\prime}$ and as $K^{\prime} \cap \partial \Omega=K^{\prime} \cap \Gamma$ we have $x_{0} \in K^{\prime} \cap \Gamma$. Hence, by (11),

$$
d\left(u(x), u\left(x_{0}\right)\right) \leq c_{7}\left|x-x_{0}\right|^{\beta} .
$$

Let $x_{1}, x_{2} \in K$. To prove Hölder continuity of $\mathrm{u}$ on $K$ it is enough to show that

$$
d\left(u\left(x_{1}\right), u\left(x_{2}\right)\right) \leq C\left|x_{1}-x_{2}\right|^{\beta} \text { if }\left|x_{1}-x_{2}\right|<\frac{1}{16} \sigma_{0},
$$

for some constant $C$. Therefore we will assume that $\sigma=\left|x_{1}-x_{2}\right|<\frac{1}{16} \sigma_{0}$. We define $\rho_{1}, \rho_{2}$ to be the distance to the boundary $\partial \Omega$ of $x_{1}, x_{2}$ respectively. We can always arrange to get $\rho_{1} \leq \rho_{2}$. Now, we consider three possible cases.

Case 1. Assume that $\rho_{1} \geq \frac{1}{16} \sigma_{0}$.

Then $x_{i} \in\left\{x \in \Omega: \operatorname{dist}(x, \partial \Omega) \geq \frac{1}{16} \sigma_{0}\right\}$ and hence from the interior Lipschitz continuity of $u$ it follows that (13) holds with some constant $C$ depending on $\sigma_{0}$, the total energy $E^{u}$ of $u$, and the metric $g$ (see [K.S.], Theorem 2.4.6).

Case 2. Assume that $\rho_{1}<\frac{1}{16} \sigma_{0}$ and $\rho_{2} \leq 2 \sigma$.

Let $y_{i} \in \partial \Omega$ be a nearest boundary point for $x_{i}$. Note that from what we assumed $\rho_{2}<\frac{1}{8} \sigma_{0}$. Therefore, (12) holds for both pairs $\left(x_{i}, y_{i}\right)$, and hence

$$
\begin{aligned}
d\left(u\left(x_{1}\right), u\left(x_{2}\right)\right) & \leq d\left(u\left(x_{1}\right), u\left(y_{1}\right)\right)+d\left(u\left(y_{1}\right), u\left(y_{2}\right)\right)+d\left(u\left(y_{2}\right), u\left(x_{2}\right)\right) \\
& \leq c_{7} \rho_{1}^{\beta}+c_{K}\left(\rho_{1}+\sigma+\rho_{2}\right)^{\alpha}+c_{7} \rho_{2}^{\beta} \\
& \leq c_{7}(2 \sigma)^{\beta}+c_{K}(5 \sigma)^{\alpha}+c_{7}(2 \sigma)^{\beta} \\
& \leq\left(4 c_{7}+5 c_{K}\right) \sigma^{\beta}
\end{aligned}
$$

and (13) holds with $C=4 c_{7}+5 c_{K}$.

Case 3. Assume that $\rho_{1}<\frac{1}{16} \sigma_{0}$ and $\rho_{2}>2 \sigma$.

Then the ball $B_{2 \sigma}\left(x_{2}\right)$ is entirely within $\Omega$. As function $x \longmapsto d^{2}\left(u(x), u\left(x_{2}\right)\right)$ is (weakly) subharmonic in $\Omega$

$$
\begin{aligned}
d^{2}\left(u\left(x_{1}\right), u\left(x_{2}\right)\right) & \leq \sup _{B_{\sigma}\left(x_{2}\right)} d^{2}\left(u(x), u\left(x_{2}\right)\right) \\
& \leq c_{8} \frac{1}{(2 \sigma)^{n-1}} \int_{\partial B_{2 \sigma}\left(x_{2}\right)} d^{2}\left(u(x), u\left(x_{2}\right)\right) d \Sigma
\end{aligned}
$$


Now we can apply (4) to the integrals over $2 \sigma$ - and $\rho_{2^{-}}$ball. This yields

$$
d^{2}\left(u\left(x_{1}\right), u\left(x_{2}\right)\right) \leq c_{8} \frac{1+O\left(\rho_{2}^{2}\right)}{1+O\left(\sigma^{2}\right)} \frac{(2 \sigma)^{2}}{\rho_{2}^{n+1}} \int_{\partial B_{\rho_{2}}\left(x_{2}\right)} d^{2}\left(u(x), u\left(x_{2}\right)\right) d \Sigma .
$$

Let $y_{2} \in \partial \Omega$ be as in Case 2. Then, for $x \in B_{\rho_{2}}\left(x_{2}\right)$ by the triangle inequality $\left|x-y_{2}\right| \leq 2 \rho_{2}$. On the other hand $\rho_{2} \leq \rho_{1}+\sigma<\frac{1}{8} \sigma_{0}$, and hence $\left|x-y_{2}\right|<\frac{1}{4} \sigma_{0}$. Therefore, from (12) and triangle inequality we estimate that for $x \in B_{\rho_{2}}\left(x_{2}\right)$

$$
\begin{aligned}
d\left(u(x), u\left(x_{2}\right)\right) & \leq d\left(u(x), u\left(y_{2}\right)\right)+d\left(u\left(x_{2}\right), u\left(y_{2}\right)\right) \\
& \leq c_{7}\left|x-y_{2}\right|^{\beta}+c_{7}\left|x_{2}-y_{2}\right|^{\beta} \\
& \leq 3 c_{7} \rho_{2}^{\beta} .
\end{aligned}
$$

We can use this estimate in (14) to get

$$
\begin{aligned}
d^{2}\left(u\left(x_{1}\right), u\left(x_{2}\right)\right) & \leq c_{9} \frac{\sigma^{2}}{\rho_{2}^{n+1}} \int_{\partial B_{\rho_{2}}\left(x_{2}\right)} d^{2}\left(u(x), u\left(x_{2}\right)\right) d \Sigma \\
& \leq c_{10}\left(\frac{\sigma}{\rho_{2}}\right)^{2} \rho_{2}^{2 \beta}=c_{10}\left(\frac{\sigma}{\rho_{2}}\right)^{2-2 \beta} \sigma^{2 \beta} \leq c_{10} \sigma^{2 \beta} .
\end{aligned}
$$

Thus (13) holds with $C=\sqrt{c_{10}}$.

This completes the proof of the Theorem in case $\alpha \neq m$. The proof for $\alpha=m$ is completely analogous.

\section{REFERENCES}

[G.S.] Gromov, M. and Schoen,R., Harmonic Maps into Singular Spaces and p-adic Superrigidity for Lattices in Groups of Rank One, IHES Publications Math. 76 (1992), 165-246.

[K.S.] Korevaar, N. and Schoen, R., Sobolev Spaces and Harmonic Maps for Metric Space Targets, To appear in Comm. Anal. Geom.

University of Utah, Salt lake City, U. S. A.

RECEIVEd SEPtEMBER 2, 1993 